\title{
Functional Recovery Patterns of Hemorrhagic and Ischemic Stroke Patients Under Post-Acute Care Rehabilitation Program
}

This article was published in the following Dove Press journal:

Neuropsychiatric Disease and Treatment

\author{
Chan-Lin Chu $\mathbb{1 D}^{1,2}$ \\ Yueh-Peng Chen ${ }^{3}$ \\ Carl CP Chen ${ }^{3,4}$ \\ Chih-Kuang Chen ${ }^{3,4}$ \\ Hsiang-Ning Chang ${ }^{4}$ \\ Chien-Hung Chang $1,2,5, *$ \\ Yu-Cheng Pei (iD) $3,4,6,7, *$
}

'Department of Neurology, Chang Gung Memorial Hospital at Linkou, Taoyuan,

Taiwan; ${ }^{2}$ School of Medicine, Chang Gung University, Taoyuan, Taiwan; ${ }^{3}$ Center for Artificial Intelligence in Medicine, Chang Gung Memorial Hospital at Linkou,

Taoyuan, Taiwan; ${ }^{4}$ Department of Physical Medicine and Rehabilitation, Chang Gung Memorial Hospital at Linkou, Taoyuan,

Taiwan; ${ }^{5}$ Department of Electrical Engineering, College of Engineering, Chang Gung University, Taoyuan, Taiwan; ${ }^{6}$ Healthy Aging Research Center, Chang Gung University, Taoyuan, Taiwan;

${ }^{7}$ Center of Vascularized Tissue Allograft, Chang Gung Memorial Hospital at Linkou, Taoyuan, Taiwan

*These authors contributed equally to this work
Correspondence: Yu-Cheng Pei;

Chien-Hung Chang

Email yspeii@gmail.com;

cva9514@gmail.com
Background: Tailored rehabilitation programs for stroke patients cannot be made without knowledge of their recovery potential. The aim of this study is to characterize the functional recovery patterns of ischemic stroke (IS) and intracerebral hemorrhage (ICH) patients under post-acute care stroke rehabilitation.

Methods: This retrospective study analyzed the data of patients enrolled in the Post-Acute Care-Cerebrovascular Disease (PAC-CVD) rehabilitation program, which provides an individualized 1- to 3-hour intensive physical, occupational, and speech and language therapy for post-acute stroke patients in Taoyuan Chang Gung Memorial hospital in Taiwan. Our primary endpoint measure was Barthel Index (BI), and secondary endpoint measures included other 12 functional measures.

Results: A total of 489 patients were included for analysis. Patients with stroke history had less BI improvement than those who suffered their first-ever stroke. In first-ever stroke patients who had completed 6 to 12 weeks of PAC-CVD program, subcortical ICH patients had greater BI, quality of life, sensation, and balance improvements, and had greater latephase recovery than their IS counterparts. In IS patients, those with age $>75$ had less BI improvement; those with National Institute of Health Stroke Scale (NIHSS) score 1-5 had greater Motor Activity Log quality of use (MAL-quality) improvement than those with NIHSS score $>5$; those with Mini-Mental State Examination (MMSE) score $\geq 24$ had greater $\mathrm{BI}$ and instrumental activities of daily living (IADL) improvement. Using the general linear model, previous stroke ( $($ : $-6.148, p=0.01)$ and subcortical ICH ( $(3: 5.04, p=0.03)$ were factors associated with BI improvement.

Conclusion: Subcortical ICH patients have greater functional improvement and greater latephase recovery than their IS counterparts following PAC rehabilitation. More studies are needed to validate our findings and unravel the underlying mechanisms of stroke recovery to optimize the treatment strategy following a stroke.

Keywords: activities of daily living, functional recovery, hemorrhagic stroke, ischemic stroke, post-acute care, rehabilitation

\section{Introduction}

Stroke causes a wide range of neurologic deficits and is the leading cause of morbidity worldwide, with a huge amount of money being expended on it. ${ }^{1,2}$ Besides acute management, rehabilitation in the subacute and chronic stages helps lessen the disability caused by stroke. Stroke can be divided into two types, intracerebral hemorrhage (ICH) and ischemic stroke (IS), with the former accounting for about $10-24 \%$ of all strokes. ${ }^{3}$ 
As most of the neurologic and functional improvements occur within the first three months after a stroke, and time-dependent recovery of body function and activities has been shown to decline significantly 10 weeks after stroke, ${ }^{6}$ the post-acute phase becomes particularly important for rehabilitative and other interventional treatments. Stroke causes a variety of functional deficits, such as cognitive and language impairment, sensorimotor dysfunction, mood disorder, and incontinence. Rehabilitation that consists of occupational therapy, which aims at improving activities of daily living (ADL), physical therapy, which aims at balance, gait, and movement, and speech and language therapy, which aims at improving communication and swallowing difficulty, has been widely implemented for post-stroke care. ${ }^{7}$

To make post-stroke rehabilitation more effective, it is better to have comprehensive functional assessment of each patient so that an individualized rehabilitation program could be provided according to patients' needs. The PostAcute Care-Cerebrovascular Disease (PAC-CVD) rehabilitation program is an innovation that initiated in 2014 by the National Health Insurance (NHI) Administration in Taiwan. Under this program, eligible patients at post-acute phase could be transferred to accredited hospitals seamlessly, where they receive comprehensive evaluation and tailored intensive rehabilitation provided by a multidisciplinary team for up to 12 weeks. This program has shown good efficacy in facilitating recovery and reducing readmission rate following a stroke. ${ }^{8-10}$

Stroke-specific and demographic factors may influence the functional outcome after stroke, such as stroke severity, ${ }^{11}$ age, ${ }^{12}$ stroke type, ${ }^{13}$ stroke location, ${ }^{14,15}$ stroke volume, ${ }^{16}$ and cognition. ${ }^{17,18}$ Previous studies showed ICH patients have a worse functional outcome than their IS counterparts, ${ }^{19}$ but the difference decreases over time and becomes non-significant after years. ${ }^{5}$

This study analyzed the data of patients enrolled in the PAC-CVD program since its initiation from March 2014 to December 2018 in a regional hospital. Patient data accessed complied with relevant data protection laws. The aim of this study is to compare the recovery patterns, including activities of daily living, swallowing function, nutrition state, sensorimotor function, quality of life (QoL), balance, mobility, cognitive and language function, between ICH and IS patients under the PAC-CVD program. Furthermore, we compared the differences in functional improvements following rehabilitation between patient subgroups stratified by post-acute care (PAC) duration, stroke type, history of stroke, stroke location, stroke severity, age, and cognitive function. We hypothesized that, following the PAC-CVD rehabilitation program, ICH patients would have better functional recovery than IS patients.

\section{Materials and Methods PAC-CVD Program}

The PAC-CVD program was initiated by the National Health Insurance Administration with the aim to improve resource allocation and patient outcome. ${ }^{10}$ Candidate patients in acute ward would first be evaluated by a case manager and may participate in the program if they meet the following criteria: (1) stroke onset time within 1 month, (2) stable hemodynamic parameters within $72 \mathrm{~h}$, (3) no neurological deterioration within $72 \mathrm{~h}$, and (4) sufficient cognitive function and ability to learn rehabilitation exercises, more specifically, with modified Rankin Scale (MRS) between 2 and 4 (between 3 and 4 since July 2017 due to the change in policy). ${ }^{8,20}$ Patient would be transferred to PAC hospital for rehabilitation, where a hospital-based multidisciplinary team, composed of a physiatrist, physical therapist, occupational therapist, speech therapist, social worker, and case manager, managed the rehabilitation program.

A formal functional assessment would be performed by team members (usually physiatrist, physical therapist, occupational therapist, and speech and language therapist) at the beginning, interim, and closing of the program; weekly meetings would be convened to report the progress, treatment plan, and goal for the patient. ${ }^{10}$

The rehabilitation consisted of a total of 3 sessions (1 session=1hour) of occupational therapy (posture training, transfers, ADL, cognitive training, constraint-induced movement therapy), ${ }^{21}$ physical therapy (balance, gait, robotic-assisted training) ${ }^{22}$ and speech and language therapy (language training, swallowing training) every weekday, depending on patients' ability. The closing (discharge) regulations are that patients showed: (1) functional improvement and the ability to receive rehabilitation in the community, (2) no functional improvement based on two consecutive functional evaluations, (3) no potential for functional recovery based on care-team evaluation, (4) patients had stayed for more than 12 weeks, (5) patients quit this program, or (6) patients died. ${ }^{10}$

PAC-CVD patients from Taoyuan Chang Gung Memorial hospital, the affiliated PAC hospital of Linkou 
Chang Gung Memorial Hospital, the largest hospital in Taiwan, were included. The protocol for this study conformed to the Helsinki Declaration and was approved by the Institutional Review Board (IRB) of Chang Gung Memorial Hospital (IRB no 201900589B0A3).

\section{Data Collection}

The data was collected from the electronic medical record, including age, sex, comorbidities (diabetes mellitus, hypertension, dyslipidemia, gout, cardiovascular disease, atrial fibrillation, kidney disease, pulmonary disease, cerebrovascular disease, liver disease, hematologic disease, malignancy, psychiatric and mood disorder, dementia), social history (smoke, alcohol), stroke onset time, stroke subtype (ICH vs IS), stroke location (cortical, subcortical, or infratentorial), side of the stroke, stroke severity, hyperacute treatment (intravenous thrombolysis, endovascular thrombectomy), complications and stroke-in-evolution at acute ward, presence of large vessel stenosis or occlusion, date of admission and discharge, various functional ability assessments (see below), cause of readmission to acute ward (if present), and discharge destination following the PAC-CVD program.

\section{Assessment of Stroke Severity and Functional Abilities}

Stroke severity at the acute care ward (almost all were neurology ward) was measured by the National Institute of Health Stroke Scale (NIHSS). In case of doubtful scoring or missing data, the admission, progress, and discharge notes of the patients would be reviewed to re-score the NIHSS according to an algorithm. This retrospective approach has shown to be reliable for stroke severity assessment. ${ }^{23,24}$ Among the $46 \mathrm{ICH}$ patients, 5 admission and 22 discharge NIHSS scores were retrospectively scored; uncertain NIHSS item scores at admission and discharge were present in $2(4 \%)$ and $9(20 \%)$ patients, respectively.

The functional abilities of patients were assessed at rehabilitation ward at four time points: on admission day, at 3 weeks, 6 weeks, and the closing of the PAC-CVD program. The assessments covered different functional domains and included the modified Rankin Scale (mRS), Barthel Index (BI), Functional Oral Intake Scale (FOIS), Mini-Nutritional Assessment (MNA), European quality of life five dimension questionnaire (Euro-QoL-5D), Instrumental activities of daily living scale (IADL), Berg Balance Scale (BBS), gait speed, 6-min walk test (6-MWT), Fugl-Meyer assessment [FuglMeyer upper extremity assessment (FuglUE), modified Fugl-Meyer sensory assessment (FuglSEN)], Mini-Mental State Examination (MMSE), Motor Activity Log amount of use (MAL-quantity), Motor Activity Log quality of use (MALquality), and Concise Chinese aphasia test (CCAT). The $\mathrm{BI}^{25}$ assesses functional independence and includes feeding, bathing, grooming, dressing, bowel control, bladder control, toileting, chair transfers, ambulation, and stair climbing. A brief description of the other scales: the mRS, ${ }^{26} \mathrm{IADL}^{27}$ and EuroQoL-5D ${ }^{28}$ give a general measurement of patient's disability; the FOIS ${ }^{29}$ measures the eating ability; the $\mathrm{MNA}^{30}$ measures the nutritional status; the BBS, ${ }^{31}$ gait speed, ${ }^{32} 6-\mathrm{MWT},{ }^{33}$ FuglMeyer assessment (FuglUE and FuglSEN), ${ }^{34}$ and MAL (MAL-quantity and MAL-quality) ${ }^{35}$ measure the motor and sensory function; the $\mathrm{MMSE}^{36}$ and $\mathrm{CCAT}^{37}$ measure the cognition and language function. Due to policy change, MMSE and MAL were no longer measured since 2018.

\section{Outcome Measures and Factor Analyses}

The primary outcome measure was the patients' BI improvement $(\triangle \mathrm{BI})$ during the PAC-CVD program; the secondary outcome measures were changes in the other functional measures mentioned above. Factors that have been shown to influence stroke outcome were analyzed, including rehabilitation duration, baseline patient characteristics, stroke type (ICH vs IS), stroke location, stroke severity, age, and cognition.

\section{Statistical Analysis}

Chi-square test was used for comparing categorical variables between groups, while Mann-Whitney test or Kruskal-Wallis test was used for continuous variables. The stroke severity and functional assessment scores of stroke subgroups were expressed as median and interquartile range (IQR). A $p$ value $\leq 0.05$ was considered statistically significant. A general linear model was used to estimate the impact of clinical variables on BI improvement. Statistical analyses were carried out using the SPSS 25.0 statistical package (SPSS, Chicago, IL).

\section{Results}

\section{Composition of the PAC-CVD Cohort}

Five-hundred and thirty-seven patients were included in the cohort (Supplementary Table S1) after excluding 35 patients who: (1) had no acute ward admission ( $n=16),(2)$ 
had onset to admission day $>5$ days $(n=7)$, for they admitted to acute ward primarily for facilitating transfer to PAC hospital, (3) had inadequate stroke assessment $(n=6)$, (4) may not actually suffer a stroke $(n=2),(5)$ had received surgical decompression due to large infarct $(n=1)$, a rare event in our cohort, (6) had subarachnoid hemorrhage $(n=1)$, (7) had subdural hemorrhage $(n=1),(8)$ was difficult to be evaluated $(\mathrm{n}=1)$.

Hyperacute treatments were performed in 24 (4.5\%) patients (intravenous thrombolysis $[\mathrm{n}=12]$, endovascular thrombectomy $[n=7]$, or both $[n=5])$. A majority $(n=476$, $88.6 \%)$ of the patients were discharged home after the PAC-CVD program; 26 (4.8\%) patients had complications during PAC that required readmission to acute ward, and $22(4.1 \%)$ patients were discharged against advice.

Four-hundred and eighty-nine patients were included for analyses after excluding 48 patients who had no assessment at the closing of the PAC program (due to against advice discharge or readmission to acute ward). The flow chart of the neurologic and functional assessments of the cohort is shown in Figure 1.

Table 1 shows the number of patients analyzed in the following parts of the study.

\section{Functional Recovery Patterns of ICH and IS Patients}

Patients Who Had No More Than 6 Weeks of PAC Had Less Severe Stroke and Made Greater Recovery Than Those Who Had 6 to 12 Weeks of PAC

Patients with up to 3 weeks ( $n=53$ ) and 3 to 6 weeks of PAC $(n=132)$ had better initial mRS and BI than those with 6 to 12 weeks of PAC $(n=304)$ (For patients with $0-3 \mathrm{w}, 3-6 \mathrm{w}$, and $6-12 \mathrm{w}$ of PAC, the mRS-I were 3 [3-4], 3 [3-4], and 4 [3-4], respectively, $p<0.01$; the BI-I were 55 [45-67], 55 [40-65], 45 [35-55], respectively, $p<0.01$ ). Patients with up to 3 weeks of PAC had greater BI improvement during the first 3 weeks than those with 3 to 6 weeks and 6 to 12 weeks of PAC (For patients with $0-3 \mathrm{w}, 3-6 \mathrm{w}$, and $6-12 \mathrm{w}$ of PAC, the $\Delta \mathrm{BI}^{0-3 \mathrm{w}}$ were 10 [5-20], 10 [5-15], 10 [0-15], respectively, $p=0.03$ ); patients with 3 to 6 weeks of PAC had greater $\mathrm{BI}$ improvement during the first 6 weeks than those with 6 to 12 weeks of PAC $\left(\triangle \mathrm{BI}^{0-6 \mathrm{w}}: 15\right.$ [10-30] vs 15 [5-20], $p<0.01)$.

When stratified by stroke type, no significant difference in BI improvement was found between ICH and IS patients $\left(\Delta \mathrm{BI}^{0-\text { final }}: 20[15-35]\right.$ vs 15 [10-25], $\left.p=0.07\right)$.

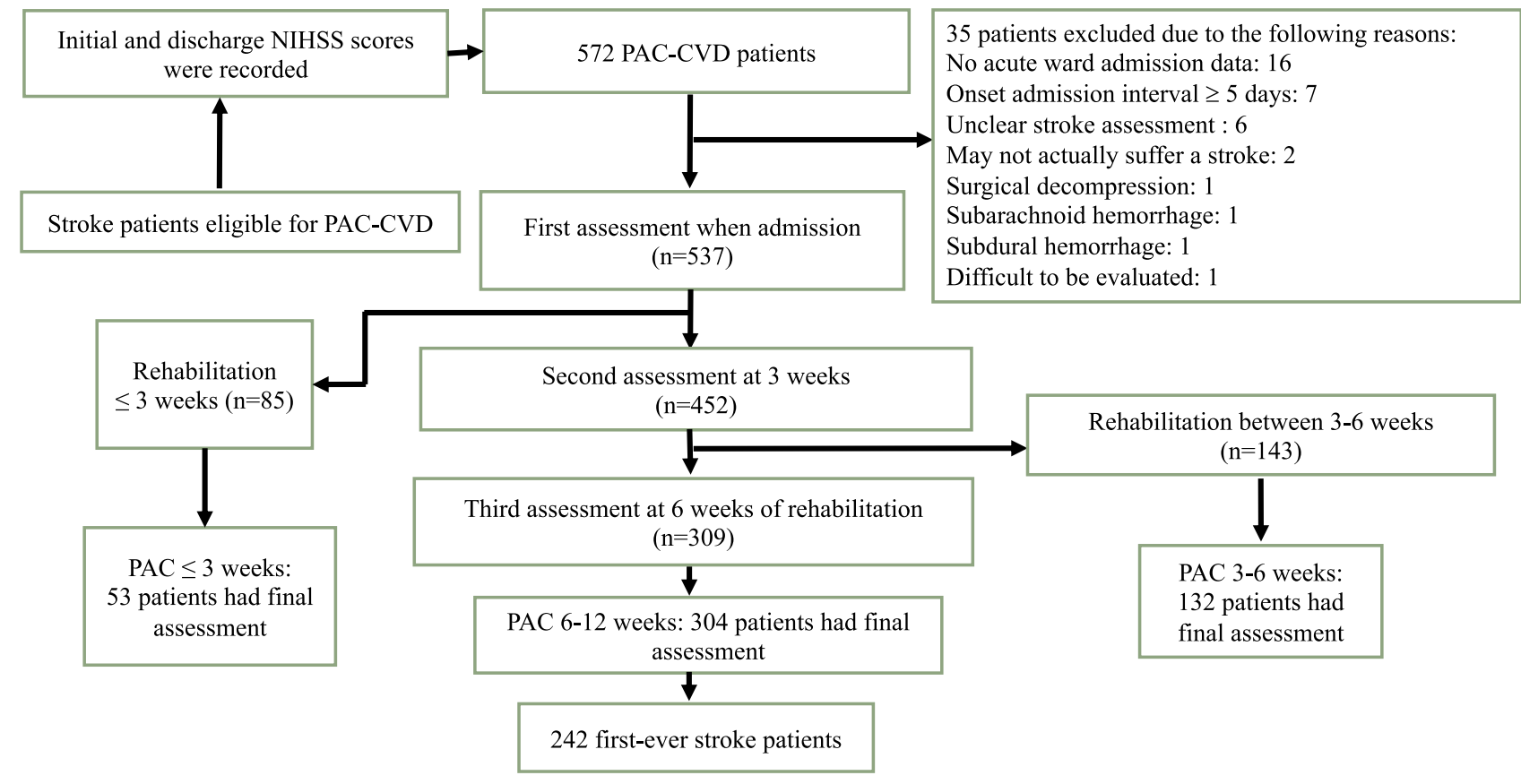

Figure I Flow chart of the neurologic and functional assessments of the patients in this study.

Abbreviations: NIHSS, National Institute of Health Stroke Scale; PAC, post-acute care; PAC-CVD, Post-Acute Care-Cerebrovascular Disease program. 
Table I Number of Patients Analyzed in Different Parts of the Study

\begin{tabular}{|c|c|c|c|}
\hline & & IS & $\mathrm{ICH}$ \\
\hline \multirow[t]{3}{*}{$\begin{array}{l}\text { Functional recovery } \\
\text { patterns of } \mathrm{ICH} \text { and IS } \\
\text { patients }\end{array}$} & 1.1 & $\begin{array}{l}44(\leq 3 w) \\
112(3-6 w) \\
258(6-12 w)\end{array}$ & $\begin{array}{l}9(\leq 3 w) \\
20(3-6 w) \\
46(6-12 w)\end{array}$ \\
\hline & 1.2 & $258(6-12 w)$ & $46(6-12 w)$ \\
\hline & $1.2 .1-1.2 .2$ & $200(6-12 w)$ & $42(6-12 w)$ \\
\hline $\begin{array}{l}\text { Factor analyses of functional } \\
\text { recovery of IS patients who } \\
\text { had } 6 \text { to } 12 \text { weeks of PAC }\end{array}$ & $\begin{array}{l}2.1 \\
2.2-2.4 \\
2.5\end{array}$ & $\begin{array}{l}258(6-12 w) \\
200(6-12 w) \\
152(6-12 w)\end{array}$ & \\
\hline
\end{tabular}

Note: Rehabilitation durations are shown in brackets.

Abbreviations: $\mathrm{ICH}$, intracerebral hemorrhage; IS, ischemic stroke.

\section{Patients Who Had 6 to I 2 Weeks of PAC}

To better obtain the functional recovery patterns of IS and ICH patients, we focused our analysis on patients who had 6 to 12 weeks of PAC, the major part in this cohort, and the results are shown in Table 2 and Supplementary Table S2.

Compared with the IS group, the ICH group had more severe stroke (NIHSS-I: 9 [6-11] vs 6 [5-9], $p<0.01$ ), and, after rehabilitation showed greater improvements in $\mathrm{BI}(\Delta \mathrm{BI}$ : 30 [20-41] vs 25 [15-35], $p=0.01$ ), QoL ( $\Delta$ Euro-QoL-5L: -3 $[-4-1]$ vs $-2[-3-1], p=0.03)$, and sensation ( $\triangle$ FuglSEN: 6 [0-18] vs $1[0-6], p<0.01)$; at the end, their $\mathrm{BI}$ and $\mathrm{QoL}$ scores were similar (BI-F: 70 [59-85] vs 70 [60-85], $p=0.81$; Euro-QoL-5L-F: 8 [7-9] vs 7 [7-9], $p=0.65)$, but the ICH group still had lower FuglSEN score (FuglSEN-F: 42 [22-$44]$ vs 44 [39-44], $p<0.01)$. There were no significant differences in changes in mRS, oral intake ability, upper limb usage, IADL, balance, or gait speed between the two groups.

\section{Patients Who Had First-Ever Stroke}

Comparison of baseline characteristics between the ICH and IS groups revealed that ICH group had a lower proportion of previous stroke ( $8.7 \%$ vs $22.5 \%, p=0.02)$. Since recurrent stroke patients make less functional gains compared with first-ever stroke patients, ${ }^{38}$ we excluded patients with previous strokes to avoid the confounding effect, and the ICH group still had greater total BI improvement than the IS group (30 [20-45] vs 25 [15-35], $p=0.02$ ); the $\mathrm{BI}$ improvement at the late phase of PAC was also greater in the $\mathrm{ICH}$ group $\left(\Delta \mathrm{BI}^{6 \mathrm{w}-\mathrm{final}}: 12.5\right.$ [5-21] vs 10 [0-20], $p=0.02$ ) (Table 3).

The following analyses in this section were performed in first-ever stroke patients.
The Subcortical Stroke Subgroup Accounted for the Greater Functional Recovery in ICH Patients

Since there was a higher proportion of subcortical type of stroke in the ICH group than their IS counterparts (73.9\% vs $45.0 \%, p<0.01$ ), and stroke location may influence the functional recovery, ${ }^{14,39}$ we stratified patients by cortical, subcortical, and infratentorial types. Among the three locations, only the subcortical ICH patients $(n=30)$ showed greater total and late phase improvement in $\mathrm{BI}(\Delta \mathrm{BI}$ : 35 [25-46] vs 30 [15-35], $\mathrm{p}=0.01 ; \Delta \mathrm{BI}^{6 \mathrm{w}-\mathrm{final}}: 15$ [5-25] vs 10 [0-20], $p=0.03$ ), sensation ( $\triangle$ FuglSEN: 9 [0-20] vs 2 [0-7], $p<0.01$ ), QoL ( $\Delta$ Euro-QoL-5D: -3 vs $-2, p=0.01$ ), and balance ( $\triangle \mathrm{BBS}: 27.5$ vs $18.5, p=0.01$ ) than their IS counterparts $(\mathrm{n}=91)$ (Table 4$)$.

We also stratified patients by right hemisphere, left hemisphere, and infratentorial subgroups ${ }^{14}$ for comparison. Left hemisphere ICH patients ( $\mathrm{n}=15,72 \%$ subcortical) showed greater $\mathrm{BI}(\triangle \mathrm{BI}: 40$ [25-45] vs 25 [15-40], $p=0.03$ ) and sensation improvement ( $\triangle$ FuglSEN: 10 [1-19] vs 1 [0-7], $p<0.01)$ than their IS counterparts $(\mathrm{n}=71,46 \%$ subcortical). These differences were not found in the right hemisphere (ICH $[\mathrm{n}=19]$ vs IS [n=61], $\Delta \mathrm{BI}: 30$ [20-45] vs 25 [15-35], $p=0.15 ; \Delta$ FuglSEN: 7 [0-22] vs $3[0-10], p=0.11)$ or infratentorial subgroups (ICH [n=8] vs IS [n=62], $\Delta$ BI: 25 [18-30] vs 25 [25-35]; $\triangle$ FuglSEN: $2[0-9]$ vs $0[0-5], p=0.29)$.

\section{Factor Analyses of Functional Recovery of IS Patients Who Had 6 to I 2 Weeks of PAC}

\section{Stroke History}

First-ever stroke patients $(\mathrm{n}=200)$ had better initial BI, and made better recovery in BI, IADL, and QoL than those with recurrent stroke $(\mathrm{n}=58)$ (BI-I: 45 [35-55] vs 42.5 [25-55], $p=0.04$; $\Delta \mathrm{BI}: 25$ [15-35] vs 20 [10-30], $p=0.02$; $\triangle \mathrm{IADL}: 1[0-2]$ vs $0[0-1], p<0.01 ; \Delta$ Euro-QoL-5D: -2 $[-3-1]$ vs $-1[-3-0], p=0.03)$. No significant difference in stroke severity between the two groups was found (6 [5-9] vs 6 [4.5-9], $p=0.6$ ).

Comparing the baseline characteristics, patients with recurrent stroke were older (Age: 71.5 [62.8-77.3] vs 64, $p<0.01$ ); no significant differences in prevalence of other comorbidities were found between the two groups.

The following analyses in this section were performed in first-ever stroke patients. 
Table 2 Comparison of IS and ICH patients who had completed 6 to 12 weeks of PAC

\begin{tabular}{|c|c|c|c|}
\hline & IS & ICH & $p$ value \\
\hline Patient number & 258 & 46 & \\
\hline Age (year) & $66(57-74)$ & $62(52-72)$ & 0.12 \\
\hline Male & $172(66.7)$ & $27(58.7)$ & 0.37 \\
\hline LOS at acute ward (days) & $13(10-17)$ & $12(10-17)$ & 0.72 \\
\hline $\begin{array}{l}\text { LOS at rehabilitation ward } \\
\text { (days) }\end{array}$ & $64(48-8 I)$ & $65(49-82)$ & 0.51 \\
\hline \multicolumn{4}{|l|}{ Comorbidities } \\
\hline Previous stroke & $58(22.5)$ & $4(8.7)$ & $0.02 *$ \\
\hline Diabetes mellitus & II $15(44.6)$ & $16(34.8)$ & 0.14 \\
\hline Hypertension & $197(76.4)$ & $34(73.9)$ & 0.42 \\
\hline Dyslipidemia & $132(5 \mid .2)$ & $18(39.1)$ & 0.09 \\
\hline Coronary artery disease & $21(8.1)$ & $7(15.2)$ & 0.11 \\
\hline Chronic kidney disease & $9(3.5)$ & $2(4.3)$ & 0.52 \\
\hline \multicolumn{4}{|l|}{ Stroke location } \\
\hline Cortical & $65(25.2)$ & $4(8.7)$ & $<0.01 *$ \\
\hline Subcortical & $116(45)$ & $34(73.9)$ & \\
\hline Infratentorial & $76(29.5)$ & $8(17.4)$ & \\
\hline \multicolumn{4}{|l|}{ Stroke severity in the acute } \\
\hline Initial NIHSS & $6(5-9)$ & $9(6-11)$ & $<0.0 I^{*}$ \\
\hline Discharge NIHSS & $5(4-7)$ & $6(4-9)$ & 0.77 \\
\hline \multicolumn{4}{|l|}{ Functional assessments in the } \\
\hline \multicolumn{4}{|l|}{ PAC hospital } \\
\hline \multicolumn{4}{|l|}{ Modified Rankin Scale } \\
\hline mRS-I & $4(3-4)$ & $4(4-4)$ & 0.15 \\
\hline mRS-F & $3(2-4)$ & $3(2-3)$ & 0.76 \\
\hline$\Delta \mathrm{mRS}$ & $0(-I-0)$ & $-I(-I-0)$ & 0.20 \\
\hline \multicolumn{4}{|l|}{ Barthel Index } \\
\hline BI-I & $45(35-55)$ & $38(25-5 I)$ & $0.03^{*}$ \\
\hline $\mathrm{BI}-\mathrm{F}$ & $70(60-85)$ & $70(59-85)$ & 0.81 \\
\hline$\Delta \mathrm{BI}$ & $25(15-35)$ & $30(20-4 I)$ & $0.01 *$ \\
\hline \multicolumn{4}{|l|}{ Euro-QoL-5D } \\
\hline Euro-QoL-5D-I & $10(9-11)$ & $10(9-12)$ & $0.05^{*}$ \\
\hline Euro-QoL-5D-F & $8(7-9)$ & $8(7-9)$ & 0.65 \\
\hline$\Delta$ Euro-QoL-5D & $-2(-3-1)$ & $-3(-4-1)$ & $0.03 *$ \\
\hline \multicolumn{4}{|l|}{ Berg Balance Scale } \\
\hline BBS-I & $25(8-25)$ & $19(5-39)$ & 0.12 \\
\hline BBS-F & $5 I(4 \mid-55)$ & $5 I(42-56)$ & 0.49 \\
\hline$\triangle \mathrm{BBS}$ & $21(11-30)$ & $26(11-37)$ & 0.10 \\
\hline \multicolumn{4}{|l|}{ Fugl-Meyer assessment } \\
\hline FuglUE-I & $44(22-56)$ & $4 \mid(5-54)$ & 0.28 \\
\hline FuglUE-F & $59(48-62)$ & $58(35-62)$ & 0.21 \\
\hline$\Delta$ FuglUE & $9(3-19)$ & $9(2-20)$ & 0.97 \\
\hline FugISEN-I & $39(30-44)$ & $31(3-42)$ & $<0.0 I^{*}$ \\
\hline FugISEN-F & $44(39-44)$ & $42(22-44)$ & $<0.01 *$ \\
\hline
\end{tabular}

(Continued)
Table 2 (Continued).

\begin{tabular}{|c|l|l|l|}
\hline & IS & ICH & $\boldsymbol{p}$ value \\
\hline$\Delta$ FugISEN & I (0-6) & $6(0-18)$ & $<0.0 I^{*}$ \\
\hline $\begin{array}{l}\text { Mini-Mental State Examination } \\
\text { MMSE-I a }\end{array}$ & $24(17-28)$ & $26(20-29)$ & 0.20 \\
MMSE-F & $29(25-30)$ & $30(26-30)$ & 0.52 \\
$\Delta$ MMSE & $3(1-6)$ & $2(0-5)$ & $0.05^{*}$ \\
\hline
\end{tabular}

Notes: Values were expressed as median (interquartile range) or counts (percentage). BBS, Berg Balance Scale; BI, Barthel Index; Euro-QoL-5D, European quality of life five dimension questionnaire; FugISEN, Fugl-Meyer sensory assessment; FuglUE, Fugl-Meyer upper extremity assessment; ICH, intracerebral hemorrhage; IS, ischemic stroke; LOS, length of stay; MMSE, Mini-Mental State Examination; mRS, modified Rankin Scale; NIHSS, National Institute of Health Stroke Scale; PAC, post-acute care. The "-l" and "-F" represent the initial and final functional assessments, respectively.

Abbreviations: ${ }^{\mathrm{a}} 193 \mathrm{Cl}$ and $30 \mathrm{ICH}$ patients were evaluated; * $\mathrm{p}$ value $\leq 0.05$.

\section{Stroke Location}

The BI improvements were similar between subgroups when stratified by either cortical, subcortical, infratentorial or hemisphere side.

\section{Age}

Less BI improvement was observed in the age $>75$ subgroup $(p=0.03)$. Specifically, the $\mathrm{BI}$ improvements were 25 [15-40], 30 [20-40], 25 [15-35], and 20 [10-35], for age $<55(n=42)$, age 55-65 $(n=59)$, age 65-75 $(n=64)$, and age $>75(n=35)$ subgroups, respectively.

\section{Stroke Severity}

Stroke severity is a crucial predictor of stroke outcome. ${ }^{40}$ Since the NIHSS score is less reflective of stroke severity in the infratentorial stroke patients, and the cut-point score of poor outcome was higher in the supratentorial than the infratentorial stroke patients (10 vs 6$),{ }^{41}$ we performed analyses for supratentorial and infratentorial stroke patients separately using different criteria.

For supratentorial stroke patients, the cut-point scores were set at 5 and 10 , as NIHSS $\leq 5$ was defined as minor stroke $^{42}$ and NIHSS $>10$ was predictive of poor outcome. $^{41}$ The NIHSS 1-5 subgroup had greater improvement in MAL-quality as compared with the other two subgroups ( $\triangle$ MAL-quality in NIHSS $1-5[\mathrm{n}=40]$, NIHSS $6-10[\mathrm{n}=46]$, and NIHSS $>10[\mathrm{n}=23]$ were 1.7 [0.4-2.4], $0.7[0.3-2.0]$, and $0.6[0-1.3]$, respectively, $p=0.02)$. The three subgroups showed comparable $\mathrm{BI}$ improvements $(\triangle \mathrm{BI}$ in NIHSS $1-5[\mathrm{n}=49]$, NIHSS $6-10$ $[\mathrm{n}=58]$, and NIHSS $>10[\mathrm{n}=31]$ were $25[20-37], 25$ [14-35], and 25 [15-35], respectively, $p=0.34$ ). 
Table 3 Comparison of Functional Measures in First-Ever IS and ICH Patients Who Had Completed 6 to 12 Weeks of PAC

\begin{tabular}{|c|c|c|c|}
\hline & IS & ICH & $p$ value \\
\hline Patient number & 200 & 42 & \\
\hline BI-I & $45(35-55)$ & $37.5(25-5 I)$ & $0.01 *$ \\
\hline $\mathrm{BI}-\mathrm{F}$ & $75(60-85)$ & $73(59-86)$ & 0.96 \\
\hline$\Delta \mathrm{Bl}^{\text {total }}$ & $25(15-35)$ & $30(20-45)$ & $0.02 *$ \\
\hline$\Delta \mathrm{Bl}^{0-3 \mathrm{w}}$ & $10(0-15)$ & $7.5(5-15)$ & 0.54 \\
\hline$\left.\Delta B\right|^{3-6 w}$ & $5(0-10)$ & $5(0-10)$ & 0.83 \\
\hline$\Delta \mathrm{BI}^{6 \mathrm{~W} \text {-final }}$ & $10(0-20)$ & $12.5(5-2 \mid)$ & $0.02 *$ \\
\hline$\Delta \mathrm{mRS}$ & $0(-I-0)$ & $-1(-1.25-0)$ & 0.18 \\
\hline$\Delta$ Euro-QoL-5D & $-2(-3-1)$ & $-2.5(-4-1)$ & 0.09 \\
\hline$\triangle \mathrm{IADL}$ & I (0-2) & I (0-2) & 0.81 \\
\hline$\Delta \mathrm{FOIS}$ & $6(5-7)$ & $6(6-7)$ & 0.57 \\
\hline$\triangle \mathrm{MNA}$ & I (0-2.5) & $2(0.6-4.1)$ & 0.34 \\
\hline$\Delta$ FugIUE & $10(3-19)$ & $9(2-20)$ & 0.97 \\
\hline$\Delta$ FugISEN & I (0-6) & $6.5(0-18)$ & $<0.01^{*}$ \\
\hline$\Delta M A L-a m o u n t^{a}$ & I $(0.2-2.1)$ & $0.75(0-1.5)$ & 0.12 \\
\hline$\triangle M A L-q u a l i t y$ & $I(0.3-2.1)$ & $0.75(0.1-1.4)$ & 0.13 \\
\hline$\triangle \mathrm{BBS}$ & 20 (II-29) & $23.5(11-37.3)$ & 0.16 \\
\hline$\Delta$ Gait-speed & $0(-3.5-10.1)$ & $4.8(-2.9-13.5)$ & 0.26 \\
\hline$\triangle 6-M W T$ & $69(40-191)$ & $58(0-151)$ & 0.29 \\
\hline$\Delta M_{M S E}{ }^{b}$ & $3(1-6)$ & $2(0-5)$ & 0.84 \\
\hline$\triangle$ CCAT $^{c}$ & $0.3(0.1-0.8)$ & $0.2(0-0.65)$ & 0.24 \\
\hline
\end{tabular}

Notes: Values were expressed as median (interquartile range) or counts. The "-I" and "-F" represent the initial and final functional assessments, respectively. ${ }^{a}$ /26 IS and $2 \mathrm{I} \mathrm{ICH}$ patients were evaluated. ${ }^{b} \mathrm{I} 52 \mathrm{IS}$ and $28 \mathrm{ICH}$ patients were evaluated. ${ }^{\mathrm{c}} \mathrm{I} 60 \mathrm{IS}$ and $30 \mathrm{ICH}$ patients were evaluated. ${ }^{*} p$ value $\leq 0.05$.

Abbreviations: 6-MWT, 6-minute walk test; BBS, Berg Balance Scale; BI, Barthel Index; CCAT, Concise Chinese aphasia test; Euro-QoL-5D, European quality of life five dimension questionnaire; FOIS, Functional Oral Intake Scale; FugISEN, FuglMeyer sensory assessment; FugIUE, Fugl-Meyer upper extremity assessment; IADL, instrumental activities of daily living; $\mathrm{ICH}$, intracerebral hemorrhage; IS, ischemic stroke; MAL-amount, Motor Activity Log amount of use; MAL-quality, Motor Activity Log quality of use; MMSE, Mini-Mental State Examination; MNA, MiniNutritional Assessment; mRS, modified Rankin Scale; PAC, post-acute care.

For infratentorial stroke patients, using a cut-point score of 6 , there was no significant difference in improvements of $\mathrm{BI}$ or other functional measures between subgroups.

\section{Cognition}

Previous studies revealed inconsistent results concerning the use of MMSE as a cognitive measure to predict the functional outcome after stroke. ${ }^{17,43}$ Using a cut-point score of $24,{ }^{36}$ the MMSE $\geq 24(\mathrm{n}=84)$ subgroup had greater improvement in $\mathrm{BI}(\Delta \mathrm{BI}: 25[15-35]$ and 20 [10-35], respectively, $p=0.05)$ and IADL ( $\triangle \mathrm{IADL}: 1$ [0-2] vs $0[0-2], p=0.04)$ and MAL-quality ( $\triangle \mathrm{MAL}-$ quality: $1.5[0.3-2.6]$ vs $0.6[0.3-1.6], p=0.02)$, as compared with the MMSE $<24(n=68)$ subgroup.

\section{General Linear Model}

Finally, a general linear model was used to evaluate the impact of clinical variables on BI improvement. Previous stroke ( $(-6.148, p=0.01)$ was associated with less improvement, and subcortical ICH ( $3: 5.04, p=0.03)$ was associated with greater improvement. Other variables, such as NIHSS and MMSE scores, age, gender, diabetes mellitus, hypertension, chronic kidney disease, and coronary artery disease, were not included in the final model.

\section{Discussion}

The PAC-CVD program in Taiwan is unique in several ways: first, it is implemented by the NHI, which covered more than $96 \%$ of Taiwan's population; Therefore, almost every stroke patient fulfilling the enrollment criteria could participate in this program; Second, the rehabilitation services are delivered by per-diem reimbursement instead of fee-for-service reimbursement, making rehabilitation more economic and efficient; ${ }^{20}$ Third, through seamless hospital transfer, it substantially shortens patients' stays in acute ward and reduces hospital expenses; Finally, patients could get the most benefit from post-stroke rehabilitation through the multidisciplinary team care and evaluation. $^{10}$

The timing of rehabilitation commencement is important in post-stroke recovery. One study showed the progress of time reflects $16 \%$ to $42 \%$ of spontaneous recovery of body functions and activities in the first 6 to 10 weeks after stroke onset, ${ }^{6}$ and early rehabilitation after stroke has been shown to correlate with better functional outcome after stroke. ${ }^{44}$ Patients in our cohort were transferred to PAC hospital at a median day of 12, and most had started neurorehabilitation when they were at acute ward.

The results of our study are consistent with previous studies that $\mathrm{ICH}$ patients showed greater functional improvements than IS patients after rehabilitation, ${ }^{5,13,45}$ and stroke location had only slight influence on ADL recovery after stroke. ${ }^{14,39}$ What our study adds is that the subcortical stroke patients account for this difference and made greater recovery at the late phase of rehabilitation than their IS counterparts. The question remains regarding the neural substrates that are responsible for this unique presentation in subcortical ICH.

Studies have shown the mechanisms of brain injury and recovery in IS and ICH are different: in IS, the mechanisms include ischemia, mass effect due to cerebral edema, and inflammation; ${ }^{46}$ In $\mathrm{ICH}$, the mechanisms additionally include the toxicity of lysed blood products on the brain parenchyma and vasculature, and the mass effect from the hematoma. ${ }^{47}$ 
Table 4 Comparison of Functional Measures of First-Ever IS and ICH Patients Who Had Completed 6 to 12 Weeks of PAC by Stroke Locations

\begin{tabular}{|c|c|c|c|}
\hline & Cortical & Subcortical & Infratentorial \\
\hline Patient number & 46,4 & 91,30 & 62,8 \\
\hline NIHSS-I & 10 vs $9.5(0.52)$ & 6 vs $9(<0.01 *)$ & 5 vs $4(0.2)$ \\
\hline BI-I & 40 vs $50(0.44)$ & 45 vs $35(<0.01 *)$ & 50 vs $60(0.16)$ \\
\hline $\mathrm{BI}-\mathrm{F}$ & 70 vs $72.5(0.85)$ & 75 vs $70(0.33)$ & 72.5 vs $85(0.28)$ \\
\hline$\Delta \mathrm{BI}^{\text {total }}$ & 20 vs $20(0.85)$ & 30 vs $35(0.01 *)$ & 25 vs $25(0.96)$ \\
\hline$\Delta \mathrm{BI}^{0-3 \mathrm{w}}$ & 10 vs $10(0.88)$ & 10 vs $5(0.52)$ & 10 vs $10(0.89)$ \\
\hline$\Delta \mathrm{Bl}^{3-6 \mathrm{w}}$ & 5 vs $5(0.52)$ & 5 vs $5(0.75)$ & 5 vs $5(0.62)$ \\
\hline$\Delta \mathrm{BI}^{6 \mathrm{w}-\text { final }}$ & 5 vs $5(0.90)$ & 10 vs $15\left(0.03^{*}\right)$ & 10 vs $10(0.53)$ \\
\hline$\Delta \mathrm{mRS}$ & 0 vs $-2(0.2)$ & 0 vs $-I(0.36)$ & $-I$ vs $-I(0.83)$ \\
\hline$\Delta$ Euro-QoL-5D & -2 vs $-0.5(0.16)$ & -2 vs $-3(0.01 *)$ & -2 vs $-2(0.78)$ \\
\hline$\triangle \mathrm{IADL}$ & 0.5 vs I $(0.74)$ & I vs I (0.9I) & I vs I (0.9I) \\
\hline$\Delta \mathrm{FOIS}$ & I vs $0\left(0.05^{*}\right)$ & I vs $0.5(0.58)$ & 0 vs $0(0.84)$ \\
\hline$\triangle \mathrm{MNA}$ & I vs $4(0.6)$ & I vs $1.75(0.73)$ & I vs $2(0.67)$ \\
\hline$\Delta$ FuglUE & 8.5 vs 8 (0.77) & II vs $10(0.61)$ & 9 vs I $(0.01 *)$ \\
\hline$\Delta$ FugISEN & 3 vs $4(0.72)$ & 2 vs $9\left(<0.0 I^{*}\right)$ & 0 vs $2(0.29)$ \\
\hline$\triangle M A L-a m o u n t^{a}$ & I.6 vs I $(0.56)$ & I. 2 vs $0.9(0.5)$ & 1.2 vs $0.15(0.16)$ \\
\hline$\Delta M A L-q u a l i t y$ & 1.6 vs $0.9(0.66)$ & 0.9 vs $0.8(0.6)$ & 1.3 vs $0.8(0.25)$ \\
\hline$\triangle \mathrm{BBS}$ & 19.5 vs $17(0.85)$ & I8.5 vs $27.5(0.01 *)$ & 22 vs $9.5(0.07)$ \\
\hline$\Delta$ Gait-speed & 0.67 vs $0.23(0.40)$ & $-I$ vs $5.56(0.05)$ & 3.13 vs $-1.89(0.66)$ \\
\hline$\Delta 6-M W T$ & 68.5 vs $49.4(0.8)$ & 55.1 vs $33.5(0.43)$ & 96 vs $125(83)$ \\
\hline$\Delta$ MMSE $^{\mathrm{b}}$ & 5 vs $2(0.13)$ & 3 vs $3(0.7 I)$ & 2 vs $0(0.06)$ \\
\hline$\triangle$ CCAT $^{c}$ & 1.3 vs $0.15(0.22)$ & 0.17 vs $0(0.74)$ & 0.05 vs $0(0.7 \mathrm{I})$ \\
\hline
\end{tabular}

Notes: Values were expressed as median ( $p$ value) or counts. The "-I" and “-F" represent the initial and final functional assessments, respectively. ${ }^{\text {In }}$ the cortical subgroup, $26 \mathrm{IS}$ and $3 \mathrm{ICH}$ patients were evaluated; in the subcortical subgroup, $6 \mathrm{I} \mathrm{IS}$ and I $3 \mathrm{ICH}$ patients were evaluated; in the infratentorial subgroup, $39 \mathrm{IS}$ and $5 \mathrm{ICH}$ patients were evaluated. 'In the cortical subgroup, $35 \mathrm{IS}$ and $4 \mathrm{ICH}$ patients were evaluated; in the subcortical subgroup, $74 \mathrm{IS}$ and $\mathrm{I} 9 \mathrm{ICH}$ patients were evaluated; in the infratentorial subgroup, $43 \mathrm{IS}$ and $5 \mathrm{ICH}$ patients were evaluated. ' In the cortical subgroup, $42 \mathrm{IS}$ and $4 \mathrm{ICH}$ patients were evaluated; in the subcortical subgroup, $75 \mathrm{IS}$ and $2 \mathrm{I} \mathrm{ICH}$ patients were evaluated; in the infratentorial subgroup, $43 \mathrm{IS}$ and $5 \mathrm{ICH}$ patients were evaluated. * $p$ value $\leq 0.05$.

Abbreviations: 6-MWT, 6-minute walk test; CCAT, Concise Chinese aphasia test; BBS, Berg Balance Scale; BI, Barthel Index; Euro-QoL-5D, European quality of life five dimension questionnaire; FOIS, Functional Oral Intake Scale; FugISEN, Fugl-Meyer sensory assessment; FuglUE, Fugl-Meyer upper extremity assessment; IADL, instrumental activities of daily living; ICH, intracerebral hemorrhage; IS, ischemic stroke; MAL-amount, Motor Activity Log amount of use; MAL-quality, Motor Activity Log quality of use; MNA, Mini-Nutritional Assessment; MMSE, Mini-Mental State Examination; mRS, modified Rankin Scale; NIHSS, National Institute of Health Stroke Scale; PAC, post-acute care.

Rehabilitation facilitates reorganization of ipsi-lesional and contra-lesional hemisphere neural network and microenvironment, including increased neuroplasticity, angiogenesis, and altered excitatory-inhibitory signaling, and augmented hematoma clearance. ${ }^{47-50}$

Advanced neuroimaging has been used to the outcome after stroke. Most of the studies applied diffusion tensor imaging (DTI), a technique that measures white matter tracts, focusing on the corticospinal tract as the outcome predictor. $^{51,52}$ A case study of a patient with right thalamic and basal ganglion ICH showed concurrent recovery of postural imbalance and recovery of medial lemniscus, which transmits proprioceptive information from the medulla to the cortex, ${ }^{53}$ highlighting the importance of sensory tracts in stroke recovery. Functional MRI study in patients with thalamic hemorrhage showed that the recovery of proprioceptive function depends on normally existing medial lemniscus and its thalamocortical pathway both ipsilateral and contralateral to the lesions. ${ }^{54}$ A recent study using MRI and voxel-based lesion symptom mapping showed lesions over corona radiata, internal capsule, insula, right inferior parietal gyrus, and left superior temporal gyrus had a higher impact on functional outcome. $^{55}$

In a rat study which ICH and IS groups were induced with comparable stroke volume and severity, ICH animals also showed better recovery of skilled walking after rehabilitation. ${ }^{56}$ In another rat study which striatal ICH was induced by collagenase, the authors concluded that the recovery of gross motor function at the early phase is caused by plasticity changes in the striatum, and skilled motor coordinate functions at the late phase are caused by changes in the motor cortex. ${ }^{57}$ In mice, axonal sprouting has been shown to correlate with motor recovery, ${ }^{58}$ but most occurs following motor recovery in their ICH counterparts. ${ }^{59}$

These findings can be accounted for by the fact that PAC rehabilitation, by augmenting the clearance of hematoma and 
toxic blood products, reactivates the previously compromised but still viable nerve fibers and makes ICH patients recover better than IS patients. At the late phase of rehabilitation, it is possible that PAC facilitates the development of alternative neural pathways that compensate for the loss of function in subcortical ICH patients. The aforementioned mechanisms will be elucidated by further clinical, imaging, and preclinical studies.

The scales used for functional measures may be responsible for the different results of studies comparing the outcomes between ICH and IS patients. Using scales that focus on the fundamental skills needed to manage basic ADL, such as BI, Rivermead Mobility Index (RMI), ${ }^{60}$ and Functional Independence Measure (FIM), ${ }^{61}$ the difference becomes significant. On the contrary, using scales, such as Mayo-Portland Adaptability Inventory-4 (MPAI-4) or IADL, ${ }^{62}$ that measure complex activities related to independent living in the community, the difference was no longer observed; In our study, changes in $\mathrm{mRS}$ were not significantly different between $\mathrm{ICH}$ and IS patients, a finding that is in contrast to that reported by Chiu et al. ${ }^{19}$ Our small sample size might account for this difference. Supplementary Table S3 summarizes the studies comparing functional outcomes between IS and ICH patients.

Following rehabilitation, both ICH and IS patients showed significant cognitive improvement, but IS patients with poorer cognitive function had less improvement in ADL. Cognitive impairment is common following a stroke, eg, slowed information processing, memory impairment, visuospatial and constructive tasks, language, arithmetic, abstract thinking, and executive function. ${ }^{63-65}$ Psychomotor speed and executive function are not specifically measured by $\mathrm{MMSE}^{66}$ but also significantly influence stroke outcomes, ${ }^{18,64,65}$ so that Montreal Cognitive Assessment (MoCA), which emphasizes on executive function and abstract thinking, may be better at predicting ADL following a stroke. ${ }^{67,68}$

There are limitations to this study: First, the relatively small ICH patient numbers limit further subgroup analysis. Second, since the eligible PAC-CVD patients were selected by mRS, selection bias is a concern when extrapolating our results in the general stroke population. There are also strengths to this study: First, a variety of functional assessments provide a valuable guide for making rehabilitation strategies; Second, the single acute care and rehabilitation hospitals avoid the heterogeneity of post-stroke care which may influence stroke outcome; Third, as far as we know, this is the largest Asian study comparing functional outcome between IS and $\mathrm{ICH}$ patients. As the etiology of stroke differs between the Asians and Western populations, eg, more small artery occlusion or intracranial atherosclerosis in the former, and more emboli from the heart or extracranial large arteries in the latter, ${ }^{69}$ it may be intriguing to know whether the conclusions drawn from the Western population studies also apply to Asians.

\section{Conclusions}

Subcortical ICH patients have greater ADL, QoL, sensation, and balance improvements and greater late-phase recovery than their IS counterparts following the PAC rehabilitation. Besides augmenting the clearance of hematoma and toxic blood products, it is possible that PAC at the late phase facilitates the development of alternative neural pathways that compensate for the loss of function in subcortical $\mathrm{ICH}$ patients. More studies are needed to validate our findings and unravel the underlying mechanisms of stroke recovery to optimize the treatment strategy following a stroke.

\section{Acknowledgment}

This project is supported by Chang Gung Medical Foundation Grants, CMRPG3B0111-3, CMRPG3G0901, CMRPG5H0051-3, and CMRPG3K0231-2 for manpower and data analysis.

\section{Funding}

This project was supported by Chang Gung Medical Foundation Grants, CMRPG3B0111-3,CMRPG3G0901, CMRPG5H0051-3, and CMRPG3K0231-2.

\section{Disclosure}

The authors declare no conflicts of interest in this work.

\section{References}

1. Johnson CO, Nguyen M, Roth GA, et al. Global, regional, and national burden of stroke, 1990-2016: a systematic analysis for the Global Burden of Disease Study 2016. Lancet Neurol. 2019;18:439-458. doi:10.1016/S1474-4422(19)30034-1

2. Katan M, Luft A. Global burden of stroke. Semin Neurol. 2018;38 (2):208-211. doi:10.1055/s-0038-1649503

3. An SJ, Kim TJ, Yoon B-W. Epidemiology, risk factors, and clinical features of intracerebral hemorrhage: an update. J Stroke. 2017;19 (1):3-10. doi:10.5853/jos.2016.00864

4. Kelly-Hayes M, Wolf PA, Kase CS, Gresham GE, Kannel WB, D'agostino RB. Time course of functional recovery after stroke: the Framingham study. J Neurol Rehabil. 1989;3(2):65-70. doi:10.1177/ 136140968900300202

5. Bhalla A, Wang Y, Rudd A, Wolfe CDA. Differences in outcome and predictors between ischemic and intracerebral hemorrhage: the South London stroke register. Stroke. 2013;44:2174-2181.

6. Kwakkel G, Kollen B, Twisk J. Impact of time on improvement of outcome after stroke. Stroke. 2006;37:2348-2353.

7. Winstein CJ, Stein J, Arena R, et al. Guidelines for adult stroke rehabilitation and recovery: a guideline for healthcare professionals from the American Heart Association/American Stroke Association. Stroke. 2016;47:e98-169. 
8. Hsieh CY, Tsao WC, Lin RT, Chao AC. Three years of the nationwide post-acute stroke care program in Taiwan. $J$ Chinese Med Assoc. 2018;81:87-88. doi:10.1016/j.jcma.2017.09.003

9. Chien SH, Sung PY, Liao WL, Tsai SW. A functional recovery profile for patients with stroke following post-acute rehabilitation care in Taiwan. J Formos Med Assoc. 2020:119;254-259.

10. Lai CL, Tsai MM, Luo JY, Liao WC, Hsu PS, Chen HY. Postacute care for stroke - A retrospective cohort study in Taiwan. Patient Prefer Adherence. 2017;11:1309-1315. doi:10.2147/PPA. S136041

11. Adams HP, Davis PH, Leira EC, et al. Baseline NIH Stroke Scale score strongly predicts outcome after stroke: a report of the Trial of Org 10172 in Acute Stroke Treatment (TOAST). Neurology. 1999;53 (1):126. doi:10.1212/WNL.53.1.126

12. Kugler C, Altenhöner T, Lochner P, Ferbert A. Does age influence early recovery from ischemic stroke? A study from the Hessian stroke data bank. J Neurol. 2003;250(6):676-681. doi:10.1007/ s00415-003-1054-8

13. Paolucci S, Antonucci G, Grasso MG, et al. Functional outcome of ischemic and hemorrhagic stroke patients after inpatient rehabilitation: a matched comparison. Stroke. 2003;34(12):2861-2865. doi:10.1161/01.STR.0000102902.39759.D3

14. Fink JN, Frampton CM, Lyden P, Lees KR. Does hemispheric lateralization influence functional and cardiovascular outcomes after stroke? An analysis of placebo-treated patients from prospective acute stroke trials. Stroke. 2008;39:3335-3340. doi:10.1161/ STROKEAHA.108.523365

15. Laredo C, Zhao Y, Rudilosso S, et al. Prognostic significance of infarct size and location: the case of insular stroke. Sci Rep. 2018;8. doi:10.1038/s41598-018-27883-3

16. Schiemanck SK, Kwakkel G, Post MWM, Prevo AJH. Predictive value of ischemic lesion volume assessed with magnetic resonance imaging for neurological deficits and functional outcome poststroke: a critical review of the literature. J Neurol Rehabil. 2006;20 (4):492-502. doi:10.1177/1545968306289298

17. Paker N, Buğdayc D, Tekdş D, Kaya B, Dere Ç. Impact of cognitive impairment on functional outcome in stroke. Stroke Res Treat. 2010

18. Mok VCT, Wong A, Lam WWM, et al. Cognitive impairment and functional outcome after stroke associated with small vessel disease. J Neurol Neurosurg Psychiatry. 2004;75(4):560-566. doi:10.1136/ jnnp.2003.015107

19. Chiu D, Peterson L, Elkind MSV, Rosand J, Gerber LM, Silverstein MD. Comparison of outcomes after intracerebral hemorrhage and ischemic stroke. $J$ Stroke Cerebrovasc Dis. 2010;19:225-229. doi:10.1016/j.jstrokecerebrovasdis.2009.06.002

20. Wang CY, Chen YR, Hong JP, Chan CC, Chang LC, Shi HY. Rehabilitative post-acute care for stroke patients delivered by per-diem payment system in different hospitalization paths: a Taiwan pilot study. Int J Qual Heal Care. 2017;29(6):779-784. doi:10.1093/intqhe/mzx102

21. Kwakkel G, Veerbeek JM, van Wegen EEH, Wolf SL. Constraintinduced movement therapy after stroke. Lancet Neurol. 2015;14 (2):224-234. doi:10.1016/S1474-4422(14)70160-7

22. Morone G, Paolucci S, Cherubini A, et al. Robot-assisted gait training for stroke patients: current state of the art and perspectives of robotics. Neuropsychiatr Dis Treat. 2017;Volume 13:1303-1311. doi:10.2147/NDT.S114102

23. Kasner SE, Chalela JA, Luciano JM, et al. Reliability and validity of estimating the NIH stroke scale score from medical records. Stroke. 1999;30(8):1534-1537. doi:10.1161/01.STR.30.8.1534

24. Williams LS, Yilmaz EY, Lopez-yunez AM. Retrospective assessment of initial stroke severity with the NIH stroke scale. Stroke. 2000;31:858-862. doi:10.1161/01.STR.31.4.858
25. Quinn TJ, Langhorne P, Stott DJ. Barthel index for stroke trials: development, properties, and application. Stroke. 2011;42:1146-1151. doi:10.1161/STROKEAHA.110.598540

26. Banks JL, Marotta CA. Outcomes validity and reliability of the modified Rankin scale: implications for stroke clinical trials: a literature review and synthesis. Stroke. 2007;38:1091-1096. doi:10.1161/01.STR.0000258355.23810.c6

27. Chong DKH. Measurement of instrumental activities of daily living in stroke. Stroke. 1995;26(6):1119-1122. doi:10.1161/01.STR.26.6.1119

28. Golicki D, Niewada M, Buczek J, et al. Validity of EQ-5D-5L in stroke. Qual Life Res. 2015;24(4):845-850. doi:10.1007/s11136-014-0834-1

29. Crary MA, Carnaby Mann GD, Groher ME. Initial psychometric assessment of a functional oral intake scale for dysphagia in stroke patients. Arch Phys Med Rehabil. 2005;86(8):1516-1520. doi:10. 1016/j.apmr.2004.11.049

30. Tsai AC, Shih CL. A population-specific mini-nutritional assessment can effectively grade the nutritional status of stroke rehabilitation patients in Taiwan. Journal of Clinical Nursing. 2009;18(1):82-88. doi:10.1111/j.1365-2702.2008.02319.x

31. Blum L, Korner-Bitensky N. Usefulness of the Berg Balance Scale in stroke rehabilitation: a systematic review. Phys Ther. 2008;88 (5):559-566. doi:10.2522/ptj.20070205

32. Richards CL, Malouin F, Dumas F, Wood-Dauphinee S. The relationship of gait speed to clinical measures of function and muscle activations during recovery post-stroke. J Biomech. 1993;26(3):324. doi:10.1016/0021-9290(93)90485-W

33. Eng JJ, Chu KS, Dawson AS, Kim CM, Hepburn KE. Functional walk tests in individuals with stroke: relation to perceived exertion and myocardial exertion. Stroke. 2002;33(3):756-761. doi:10.1161/ hs 0302.104195

34. Sullivan KJ, Tilson JK, Cen SY, et al. Fugl-Meyer assessment of sensorimotor function after stroke. Stroke. 2011;42(2):427-432. doi:10.1161/STROKEAHA.110.592766

35. Uswatte G, Taub E, Morris D, Vignolo M, McCulloch K. Reliability and validity of the upper-extremity motor activity log-14 for measuring real-world arm use. Stroke. 2005;36(11):2493-2496. doi:10.1161/ 01.STR.0000185928.90848.2e

36. Bour A, Rasquin S, Boreas A, Limburg M, Verhey F. How predictive is the MMSE for cognitive performance after stroke? J Neurol. 2010;257:630-637. doi:10.1007/s00415-009-5387-9

37. Zhong YM, Li SE, Zhang MX. Concise Chinese Aphasia test. Taipei: Psychological Publishing Co, Ltd; 2003. Available from: https://scho lar.google.com/scholar_lookup?title=Concise Chinese Aphasia Test\&author=Y. Zhong\&publication_year=2003.

38. Ng YS, Tan KHX, Chen C, Senolos GC, Koh GCH. How do recurrent and first-ever strokes differ in rehabilitation outcomes? $\mathrm{Am}$ $J$ Phys Med Rehabil. 2016;95(10):709-717. doi:10.1097/ PHM.0000000000000502

39. Halkes PHA, Kappelle LJ, Van Gijn J, Van Wijk I, Koudstaal PJ, Algra A. Large subcortical infarcts: clinical features, risk factors, and long-term prognosis compared with cortical and small deep infarcts. Stroke. 2006;37:1828-1832. doi:10.1161/01.STR.0000226993.88307.ff

40. Rost NS, Bottle A, Lee JM, et al. Stroke severity is a crucial predictor of outcome: an international prospective validation study. $J$ Am Heart Assoc. 2016;5:1-7. doi:10.1161/JAHA.115.002433

41. Yoshimura S, Lindley RI, Carcel C, et al. NIHSS cut point for predicting outcome in supra- vs infratentorial acute ischemic stroke. Neurology. 2018;91:E1695-701. doi:10.1212/WNL.0000000000006 437

42. Khatri P, Kleindorfer DO, Devlin T, et al. Effect of alteplase vs aspirin on functional outcome for patients with acute ischemic stroke and minor nondisabling neurologic deficits the PRISMS randomized clinical trial. JAMA - J Am Med Assoc. 2018;320:156-166. doi:10.1001/jama.2018.8496 
43. Özdemir F, Birtane M, Tabatabaei R, Ekuklu G, Kokino S. Cognitive evaluation and functional outcome after stroke. Am J Phys Med Rehabil. 2001;80:410-415. doi:10.1097/00002060-200106000-00003

44. Maulden SA, Gassaway J, Horn SD, Smout RJ, DeJong G. Timing of initiation of rehabilitation after stroke. Arch Phys Med Rehabil. 2005;86(12):34-40. doi:10.1016/j.apmr.2005.08.119

45. Chae J, Zorowitz RD, Johnston MV. Functional outcome of hemorrhagic and nonhemorrhagic stroke patients after in-patient rehabilitation. Am J Phys Med Rehabil. 1996;75(3):177-182. doi:10. 1097/00002060-199605000-00004

46. Deb P, Sharma S, Hassan KM. Pathophysiologic mechanisms of acute ischemic stroke: an overview with emphasis on therapeutic significance beyond thrombolysis. Pathophysiology. 2010;17:197-218. doi:10.1016/ j.pathophys.2009.12.001

47. Benowitz LI, Carmichael ST. Promoting axonal rewiring to improve outcome after stroke. Neurobiol Dis. 2010;37(2):259-266. doi:10.1016/j.nbd.2009.11.009

48. Alia C, Spalletti C, Lai S, et al. Neuroplastic changes following brain ischemia and their contribution to stroke recovery: novel approaches in neurorehabilitation. Front Cell Neurosci. 2017;11. doi:10.3389/ fncel.2017.00076

49. Angels Font M, Arboix A, Krupinski J. Angiogenesis, neurogenesis and neuroplasticity in ischemic stroke. Curr Cardiol Rev. 2010;6:238-244.

50. Williamson MR, Dietrich K, Hackett MJ, et al. Rehabilitation augments hematoma clearance and attenuates oxidative injury and ion dyshomeostasis after brain hemorrhage. Stroke. 2017;48:195-203.

51. Moura LM, Luccas R, De Paiva JPQ, et al. Diffusion tensor imaging biomarkers to predict motor outcomes in stroke: a narrative review. Front Neurol. 2019;10:445.

52. Chaudhary N, Pandey AS, Gemmete JJ, et al. Diffusion tensor imaging in hemorrhagic stroke. Exp Neurol. 2015;272:88-96.

53. Jang SH, Do Lee H. Recovery of an injured medial lemniscus with concurrent recovery of pusher syndrome in a stroke patient: a case report. Med. 2018;97.

54. Lee MY, Kim SH, Choi BY, Chang CH, Ahn SH, Jang SH. Functional MRI finding by proprioceptive input in patients with thalamic hemorrhage. NeuroRehabilitation. 2012;30:131-136.

55. Cheng B, Forkert ND, Zavaglia M, et al. Influence of stroke infarct location on functional outcome measured by the modified Rankin Scale. Stroke. 2014;45:1695-1702.

56. Mestriner RG, Netto CA, Miguel PM, et al. Behavior outcome after ischemic and hemorrhagic stroke, with similar brain damage, in rats. Behav Brain Res. 2013;244:82-89.
57. Tamakoshi K, Ishida A, Takamatsu Y, et al. Motor skills training promotes motor functional recovery and induces synaptogenesis in the motor cortex and striatum after intracerebral hemorrhage in rats. Behav Brain Res. 2014;260:34-43.

58. Overman JJ, Clarkson AN, Wanner IB, et al. A role for ephrin-A5 in axonal sprouting, recovery, and activity-dependent plasticity after stroke. Proc Natl Acad Sci U S A. 2012;109:E2230-9.

59. Barratt HE, Lanman TA, Carmichael ST. Mouse intracerebral hemorrhage models produce different degrees of initial and delayed damage, axonal sprouting, and recovery. J Cereb Blood Flow Metab. 2014;34:1463-1471.

60. Collen FM, Wade DT, Robb GF, Bradshaw CM. The rivermead mobility index: a further development of the Rivermead motor assessment. Int Disabil Stud. 1991;13:50-54.

61. Mackintosh S. Functional independence measure. Aust J Physiother. 2009;55:65.

62. Mlinac ME, Feng MC. Assessment of activities of daily living, self-care, and independence. Arch Clin Neuropsychol. 2016;31:506-516.

63. Hsu Y, Chen G, Chen P, Lin S. Postacute care model of stroke in one hospital. Tzu Chi Med J. 2019;31:260-265.

64. Perna R, Temple J. Rehabilitation outcomes: ischemic versus hemorrhagic strokes. Behav Neurol. 2015;2015.

65. Kelly PJ, Furie KL, Shafqat S, Rallis N, Chang Y, Stein J. Functional recovery following rehabilitation after hemorrhagic and ischemic stroke. Arch Phys Med Rehabil. 2003;84:968-972.

66. Jørgensen HS, Nakayama H, Raaschou HO, Olsen TS. Intracerebral hemorrhage versus infarction: stroke severity, risk factors, and prognosis. Ann Neurol. 1995;38:45-50.

67. Franke CL, Van Swielen JC, Algra A, Van Gijn J. Prognostic factors in patients with intracerebral haematoma. J Neurol Neurosurg Psychiatry. 1992;55:653-657.

68. Hochstenbach J, Mulder T, Van Limbeek J, Donders R. Cognitive decline following stroke: a comprehensive study of cognitive decline following stroke. J Clin Exp Neuropsychol. 1998;20: 503-517.

69. Barker-Collo S, Krishnamurthi R, Feigin V, et al. Neuropsychological outcome and its predictors across the first year after ischaemic stroke. Brain Impair. 2016;17:111-122.
Neuropsychiatric Disease and Treatment

\section{Publish your work in this journal}

Neuropsychiatric Disease and Treatment is an international, peerreviewed journal of clinical therapeutics and pharmacology focusing on concise rapid reporting of clinical or pre-clinical studies on a range of neuropsychiatric and neurological disorders. This journal is indexed on PubMed Central, the 'PsycINFO' database and CAS, and
Dovepress

is the official journal of The International Neuropsychiatric Association (INA). The manuscript management system is completely online and includes a very quick and fair peer-review system, which is all easy to use. Visit http://www.dovepress.com/testimonials.php to read real quotes from published authors. 